\title{
Ultra-Narrow Laser for Optical Frequency Reference
}

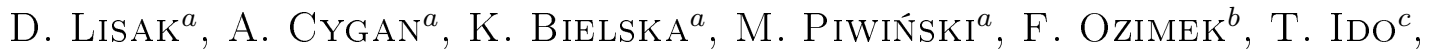 \\ R.S. TRAWińsKi ${ }^{a}$ AND R. CiURYŁO ${ }^{a}$ \\ ${ }^{a}$ Instytut Fizyki, Uniwersytet Mikołaja Kopernika, Grudziądzka 5/7, 87-100 Toruń, Poland \\ ${ }^{b}$ Instytut Fizyki Doświadczalnej, Wydział Fizyki, Uniwersytet Warszawski, Hoża 69, 00-681 Warszawa, Poland \\ ${ }^{c}$ National Institute of Information and Communications Technology, Koganei, Tokyo 184-8795, Japan
}

(Received December 21, 2011)

\begin{abstract}
We present ultra-narrow line width, tunable diode laser system which will be used as a local oscillator in an optical atomic clock and for precision spectroscopy of Sr near $689 \mathrm{~nm}$. Design of the high finesse optical cavity used as short-term frequency reference is optimized with respect to insensitivity to vibrations. We achieved laser line width of about $8 \mathrm{~Hz}$, measured by comparison of two identical systems. The relative phase lock of two lasers is better than $150 \mathrm{mHz}$. Laser tunability and usefulness for precison spectroscopy were demonstrated through line shape measurement of a $20 \mathrm{kHz}$ wide resonance of the optical cavity.
\end{abstract}

PACS: $42.60 . \mathrm{By}, 42.62 . \mathrm{Eh}$

\section{Introduction}

The dynamic development of semiconductor laser technology has resulted in creation of sophisticated tunable laser systems having ultra low spectral line width for a reasonable cost. In 1988 it was demonstrated by Salomon et al. [1] that the frequency of laser light can be locked to a resonant frequency of an optical cavity with a precision exceeding $0.1 \mathrm{~Hz}$. Since then, construction of stable, high-finesse optical cavity is a key to the construction of lasers characterized by a short-term stability better than $1 \mathrm{~Hz}$ [2]. Research on the design and mounting of optical cavities led to a significant simplification of such systems and reduced their vulnerability to external environment in the laboratory [3-6]. This gives the opportunity to design a relatively simple and small systems with ultra-stable frequency of generated laser radiation and spectral width of the order of $1 \mathrm{~Hz}[7-10]$.

These types of laser systems are successfully used in precision studies of narrow resonances, such as those in the ultra-cold strontium close to the ${ }^{1} S_{0}{ }^{3} P_{1}$ intercombination transition [11-14]. Moreover, in combination with ultra-narrow atomic resonances they are crucial element of optical clocks [15-20].

In this paper we present an ultra-stable laser system built in the National Laboratory FAMO in Torun, Poland. Experimental setup is built with almost only commercially available elements. Tests of the system stability and tunability are presented. Laser will be used as a local oscillator in Sr optical clock system. Its broad and precise tunability will allow to use it for spectroscopy with sub $\mathrm{kHz}$ resolution. Moreover, the laser system can serve to stabilize a frequency axis for our cavity-enhanced molecular spectroscopy experiments $[21,22]$.

\section{Optical cavity}

Design of the optical cavity is critical for efficient laser line width narrowing and its frequency stabilization. With good Pound-Drever-Hall (PDH) lock one can achieve line width reduction by a factor of about $10^{3}-10^{4}$ compared to the width of the cavity resonance to which laser is locked, see e.g. [2, 9]. Our cavity is built with two mirrors having nominal reflectivity $R \approx 0.99995$ for a wavelength range 689-698 nm. Both mirrors and the spacer are made of ultra-low expansion (ULE) glass. Mirrors are optically contacted to a $100 \mathrm{~mm}$ spacer, thus free spectral range (FSR) of the cavity is $\nu_{\mathrm{f}}=1.499 \mathrm{GHz}$ and the nominal full width at half maximum (FWHM) of the cavity optical resonance is $\delta \nu=\nu_{\mathrm{f}} / F^{*}=\nu_{\mathrm{f}}(1-R) /(\pi \sqrt{R}) \approx 23.9 \mathrm{kHz}$, where $F^{*} \approx 62800$ is the designed cavity finesse. This value of $\delta \nu$ is small enough to narrow the laser line width even to a hertz level with sufficiently fast and low-noise feedback loop.

\subsection{Optimization of the cavity shape}

The achievable width of the laser is dependent on stability of the optical cavity. The frequency of each cavity mode is dependent on the optical length of the cavity. Assuming that the cavity is in vacuum the effective optical path length will depend on mirrors separation and angle between them. The ultimate limit of the frequency stability of the cavity mode is determined by thermal noise dependent on the shape of the cavity and the material from which it is made. This problem has been elaborated theoretically and experimentally in several papers [23-25]. Optimization of the cavity shape with respect to its insensitivity to mechanical vibrations was done using finite element method (FEM) in Refs. [3-6]. In these 
works influence of the change of cavity shape, tilt and deformation of mirrors caused by acceleration was carefully analyzed.

Simple model demonstrating how tilt of the mirrors changes the frequency of the cavity mode can be given assuming no deformation of mirrors. In Fig. 1 we show relation between tilt of mirrors and effective length $Y$ of the resonator assuming that separation of mirror centers was not changed.

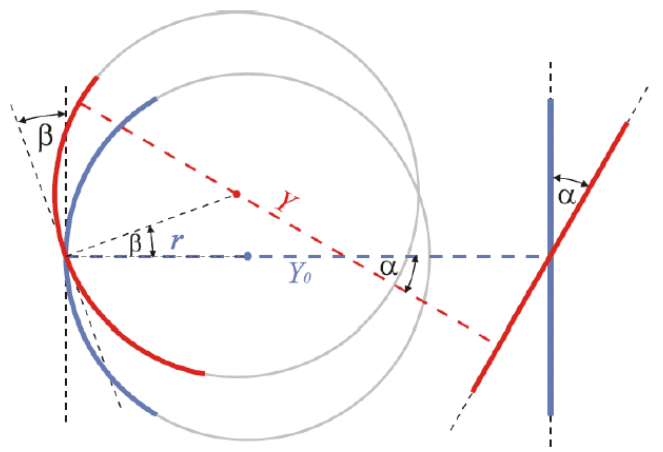

Fig. 1. Influence of the cavity mirrors tilt on effective length of the resonator. See in text for details.

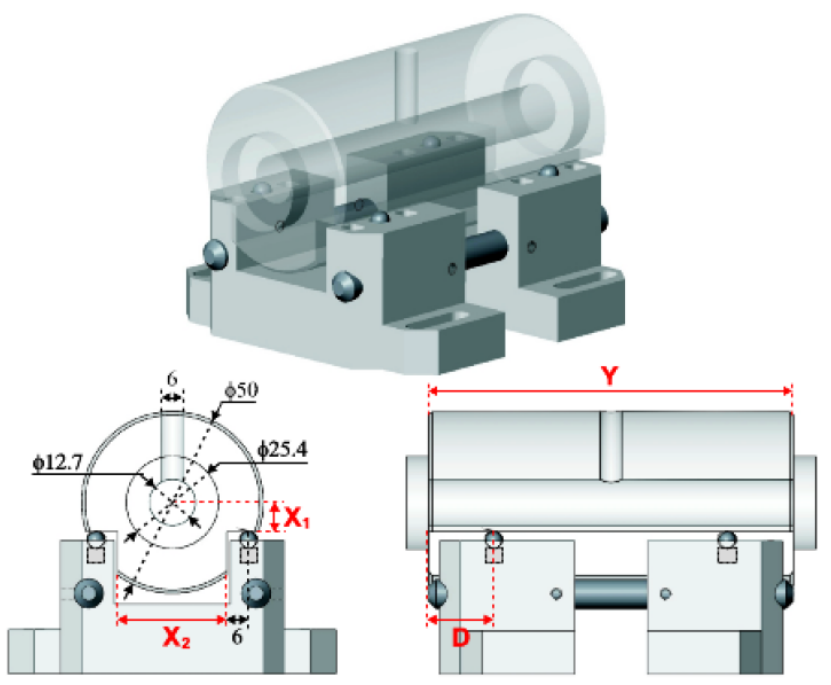

Fig. 2. Design and dimensions (in $\mathrm{mm}$ ) of the optical cavity and its support. See in text for details.

In our case one of mirrors is spherical and the other is flat. It is easy to show that

$$
Y=Y_{0} \cos \alpha+r(1+\sin \alpha \sin \beta-\cos \beta \cos \alpha),
$$

where $Y_{0}$ is initial separation of mirror centers, $r$ is radius of curvature of the mirror, $\alpha$ is tilt angle of the flat mirror and $\beta$ is tilt angle of the spherical mirror. For very small angles our expression can be simplified

$$
Y=Y_{0}-\frac{Y_{0}}{2} \alpha^{2}+\frac{r}{2}(\alpha+\beta)^{2} .
$$

As one can see, change of the effective length of resonator depends on angles in square. Therefore in our case it will have no significant influence on the effective length $Y$ of the resonator. The most important is to take a proper care of stability of initial separation $Y_{0}$ between mirrors.

Shape of the cavity and its points of support were carefully modeled using finite element method (FEM) to minimize sensitivity of the cavity optical resonance frequencies to mechanical vibrations. In Fig. 2 we present drawings and dimensions of the cavity and its support. A basic concept of the cavity shape is adopted from Webster et al. [5]. FEM analysis available in SolidWorks software was used to find optimal combination of dimensions of the cutouts along the spacer (dimensions $X_{1}, X_{2}$ ) and of 4 symmetrical support points $D$. Similar kind of analysis for horizontal cavities were done by Nazarova et al. [3] and by Webster et al. [5]. Also vertical cavities [9] were investigated in this way. Modeling results obtained by Webster et al. [5] were in relatively good agreement with experimental data [5].

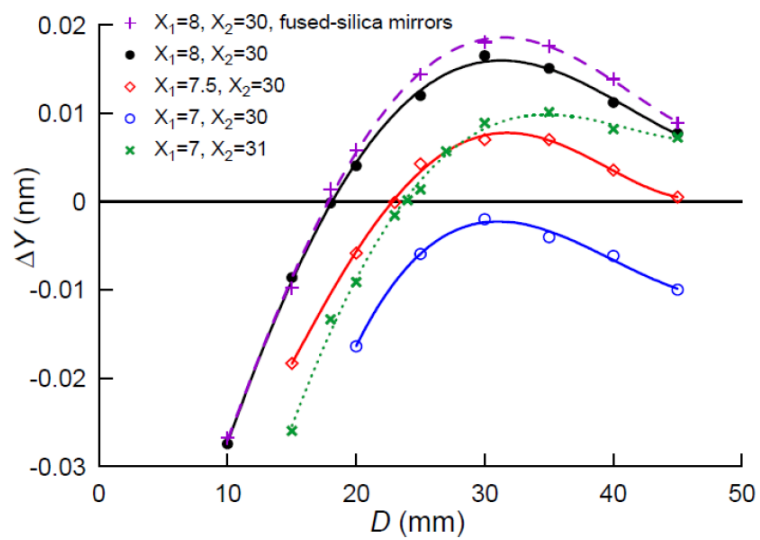

Fig. 3. Change in cavity length $\Delta Y$ as a function of support point distance $D$ from the spacer edge for different configurations of the cavity.

We chose the design proposed by Webster et al. [5] because it allows easy tuning of the cavity sensitivity to vibrations by changing points of support. We should note that in typical laboratory conditions for horizontally mounted cavity the most troublesome vibrations are these in vertical direction [3].

In Fig. 3 change in cavity length $\Delta Y$ is presented as a function of support point $D$ distance from the spacer edge for different configurations of the cavity. All presented data are calculated for vertical acceleration $a=10 \mathrm{~m} / \mathrm{s}^{2}$. Quantity $\Delta Y$ is defined as a difference of distance between mirror centers $Y$ with acceleration $a$ and without it $\Delta Y=Y(a)-Y(0)$. In order to make FEM calculations one needs to know mechanical parameters of the material. Both the spacer and mirrors were made with a special ULE glass. Physical properties of this glass can be found in [26], the Young modulus $E=67.6 \times 10^{9} \mathrm{~Pa}$ and Poisson's ratio $\nu=0.17$. As shown in Fig. 3 for $X_{1}=8 \mathrm{~mm}, X_{2}=30 \mathrm{~mm}$, support points can be placed at such distance $D$ (about $18 \mathrm{~mm}$ ) that $\Delta Y=0$, which 
means insensitivity of cavity length to vertical acceleration $a$. The optimal support point $D$ can be shifted by slightly varying dimensions $X_{1}$ or $X_{2}$ of the spacer cutouts. However for $X_{1}=7 \mathrm{~mm}$ and $X_{2}=30 \mathrm{~mm}$ insensitivity of $Y$ on $a$ cannot be achieved for any $D$. The most convenient configuration would be to have minimal derivative $\mathrm{d} Y / \mathrm{d} D$ at point where $\Delta Y$ crosses zero. Such result should occur for $7<X_{1}<7.5, X_{2}=30 \mathrm{~mm}$ and in theory should assure the highest tolerance on positioning the cavity on its support. On the other hand, systematical error of our FEM calculations is difficult to estimate. Except for numerical errors associated with finite numbers of elements and precision of computations one should consider imperfect material properties and small differences of dimensions between model and the real spacer. For example we calculated another curve in Fig. 3 for $X_{1}=7 \mathrm{~mm}, X_{2}=30 \mathrm{~mm}$ but with mirrors made of fused silica instead of ULE glass. Difference between $\Delta Y$ for these two configurations are up to $10 \%$ at point of $\mathrm{d} Y / \mathrm{d} D=0$. In order to increase tolerance for systematical error of simulations and to assure occurrence of point of insensitivity to $a$ we finally chose a compromise solution with $X_{1}=8 \mathrm{~mm}$ and $X_{2}=30 \mathrm{~mm}$.

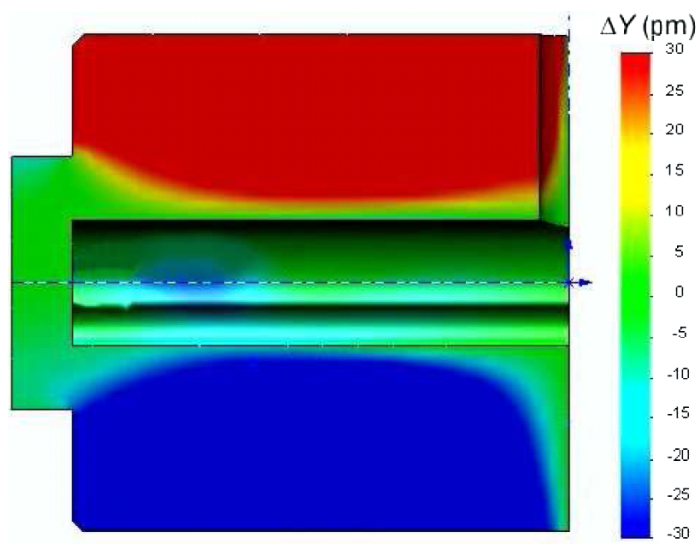

Fig. 4. FEM simulation of a local displacement in the cavity horizontal axis direction under vertical acceleration $a=10 \mathrm{~m} / \mathrm{s}^{2}$

In Fig. 4 a cross-section of the cavity is presented with color map corresponding to a local displacement in the cavity horizontal axis direction under vertical acceleration $a=10 \mathrm{~m} / \mathrm{s}^{2}$. Cavity symmetry allows to simulate only a quarter of the cavity. Calculations presented in Fig. 4 were made for the optimal configuration and it is clearly seen that the mirror center has minimal displacement while upper and lower parts of the spacer move in the opposite directions.

Change $\Delta Y$ of the mirror-to-mirror distance $Y$ can be converted to change $\Delta \nu_{\mathrm{c}}$ of the cavity mode frequency $\nu_{\mathrm{c}}$ using relation $\Delta Y / Y=-\Delta \nu_{\mathrm{c}} / \nu_{\mathrm{c}}$. Assuming that the cavity can be placed on support in optimal position $D$ with precision of $\pm 1 \mathrm{~mm}$ we can estimate expected sensitivity of $\nu_{\mathrm{c}}$ to the cavity vertical acceleration $a$. From Fig. 3 near $D=18 \mathrm{~mm}$ deriva- tive $\mathrm{d}(\Delta Y) / \mathrm{d} D=2.4 \times 10^{-9}$, which for $1 \mathrm{~mm}$ displacement of the cavity from optimal $D$ gives sensitivity $\Delta Y / a=2.4 \times 10^{-13} \mathrm{~m} /\left(\mathrm{m} / \mathrm{s}^{2}\right)$. For cavity mode frequency $\nu_{\mathrm{c}}=440 \mathrm{THz}$ we estimate sensitivity to vertical acceleration $\Delta \nu_{\mathrm{c}} / a=1.1 \mathrm{kHz} /\left(\mathrm{m} / \mathrm{s}^{2}\right)$. This is comparable to experimental result of Nazarova et al. [3] $\left(1.5 \mathrm{kHz} /\left(\mathrm{m} / \mathrm{s}^{2}\right)\right)$ for their FEM-optimized cavity. Typical order of magnitude of acceleration in laboratory conditions with well isolated cavity is $10^{-5} \mathrm{~m} / \mathrm{s}^{2}[3]$. Assuming the same number for our cavity with isolation system described in the next section, mode frequency shifts $\Delta \nu_{\mathrm{c}}$ caused by vertical acceleration should be below $20 \mathrm{mHz}$ for our cavity.

\subsection{Mechanical and acoustic isolation}

As discussed above even for cavity with optimized shape, dependence of cavity mode frequency on acceleration caused by mechanical and acoustic noise cannot be completely eliminated. Therefore cavity should be isolated from laboratory environment. In Fig. 5 our cavity isolation system is presented. Cavity is placed horizontally on 4 stainless steel balls which touch the cavity at optimal points calculated as described earlier (see also Fig. 2). Aluminum support has square sockets for the balls with $4 \mathrm{~mm}$ thick viton pads on the bottom. Viton, except of damping vibrations, assures equal distribution of load to all 4 balls, which otherwise could be perturbed by slightly unequal depth of sockets. The cavity support consists of 2 equal parts connected by steel rods which allow to vary the distance between points of cavity support in order to fine-tune its optimal value. Cavity with support is closed in all-metal vacuum chamber equipped with ion pump. Design of the chamber is identical with that used in Ref. [27]. Evacuation of the cavity improves its resonance frequencies stability for three reasons. Passive thermal stability is higher because of elimination of thermal conductivity between cavity and chamber. Although coefficient of thermal expansion (CTE) of ULE glass is very small (below $3 \times 10^{-8} / \mathrm{K}$ at room temperature) we will show later that temperature change is dominant source of frequency drift of our laser. Vacuum chamber also reduces influence of acoustic noise which can only be transmitted to the cavity through its support. Finally absence of any gas between mirrors eliminates problem of its refractive index variations with pressure. The vacuum chamber is placed inside another aluminum chamber in order to minimize its temperature gradients and it is placed on $5.5 \mathrm{~cm}$ thick aluminum optical breadboard. The main mechanical isolation is provided by vibration isolation platform (Minus-k, BM-1) on which the optical breadboard is placed. Between the platform and the breadboard additional steel plate is placed to increase the platform's load to its optimal value. Steel plate is equipped with viton dumpers distributed proportionally to their loads. The vibration isolation platform eliminated both horizontal and vertical vibrations with frequencies higher than $0.5 \mathrm{~Hz}$. Further elimination of acoustic noise, as well as improvement of thermal stability, is realized by placing the platform inside heavy 
steel chamber lined with acoustic-damping foam (Novascan NanoCube). According to its specifications it reduces acoustic noise by up to $40 \mathrm{~dB}$. The whole system is placed on a standard optical table.

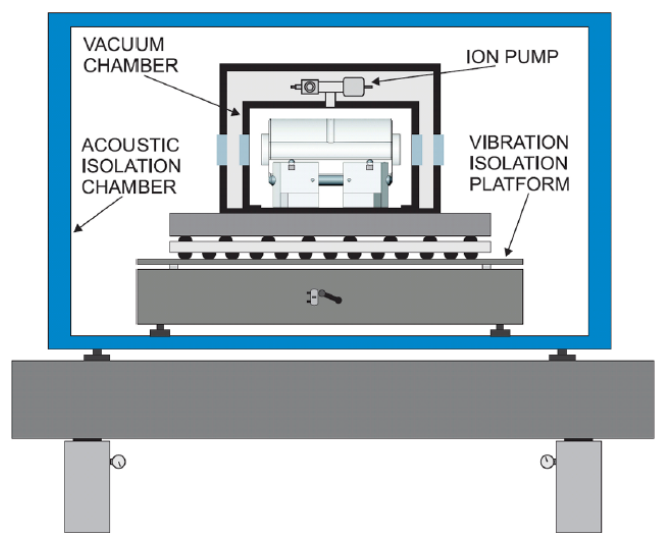

Fig. 5. System of thermal, mechanical and acoustic isolation of the optical cavity. See in text for details.

\section{Experimental setup}

Our laser system is based on commercially available external cavity diode lasers, ECDL (Toptica DL-pro and DL-100) which both provide about $20 \mathrm{~mW}$ output power at wavelength $687-693 \mathrm{~nm}$. Their spectral line widths measured at $5 \mu$ s time period are $<200 \mathrm{kHz}$ for DL-pro and $<1 \mathrm{MHz}$ for DL-100.

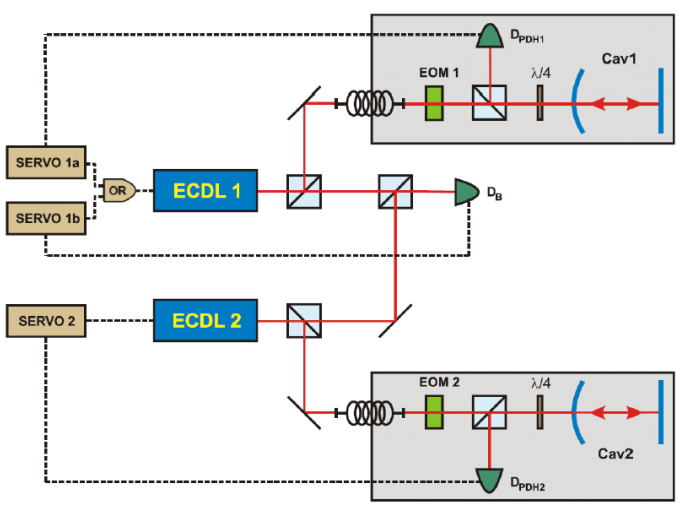

Fig. 6. Scheme of experimental setup for independent lock of two lasers (ECDL 1 and ECDL 2) to optical cavities (Cav1 and Cav2), and for phase lock of laser ECDL 1 to ECDL 2.

In Fig. 6 scheme of experimental setup is presented. ECDL 1 and ECDL 2 are DL-pro and DL-100 laser heads, respectively. Each of them can be independently locked to its own identical optical cavity (Cav1 and Cav2) with use of the PDH locking method. Detailed description of the PDH method can be found e.g. in Refs. $[28,29]$. Laser beam from ECDL1 is delivered through polarization-maintaining single-mode fiber to the optical breadboard isolated from mechanical and acoustic noise as described in the previous section. Laser light is phase-modulated (PM) with electro-optical modulator (EOM1) at frequency $\Omega=20 \mathrm{MHz}$, and beam is directed into the reference optical cavity (Cav1). Shape of the beam is mode-matched to the $\mathrm{TEM}_{00}$ mode of the cavity with a set of two lenses. Light reflected from- and leaking out of the cavity is directed to the $125 \mathrm{MHz}$ bandwidth Si detector $\left(\mathrm{D}_{\mathrm{PDH} 1}\right)$. The antisymmetric PDH error signal can be extracted from $\mathrm{D}_{\mathrm{PDH} 1}$ detector signal demodulated at frequency $\Omega$ by mixer (Mini-Circuits ZP-3), see Refs. [28, 29] for underlying PDH locking theory. The error signal is used in a feedback loop that controls laser frequency presented in Fig. 7a. As a loop filter we use fast PID controller (Toptica FALC) which has maximum bandwidth of $10 \mathrm{MHz}$. Its main output signal controls the laser diode current through fast modulation input of the laser head. Additionally, slow unlimited integrator, available in FALC, is used to tune laser frequency by changing its diffraction grating angle. This second stage of the feedback loop has much lower bandwidth (a few $\mathrm{kHz}$ ) but higher dynamic range than diode current modulation. Its purpose is to respond to slow laser frequency drift and keep the DC component of the current modulation signal close to zero.
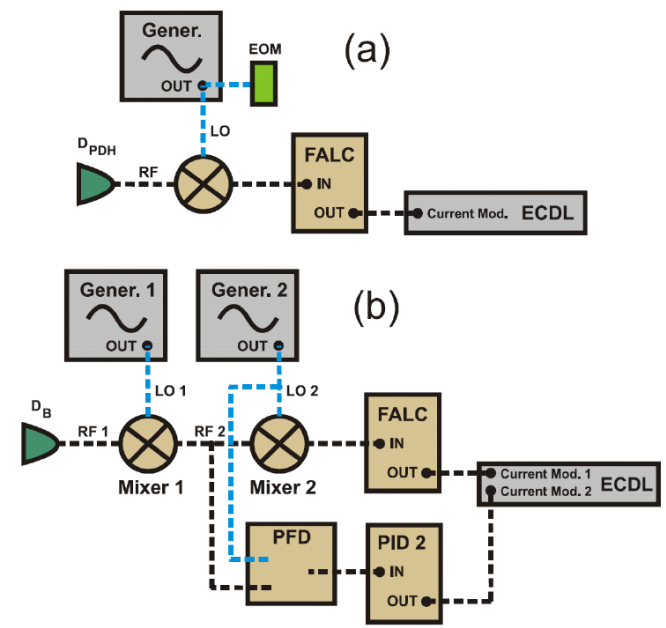

Fig. 7. Feedback loop scheme for: (a) PDH laser lock to the optical cavity, (b) phase-lock of one laser to another. See in text for details.

Similar setup is built for ECDL2 laser locking to Cav2 optical cavity. With this setup optical beat note of two independently locked lasers can be measured. Laser beams of both lasers interfere on fast Si detector $\mathrm{D}_{\mathrm{B}}$ with bandwidth of $20 \mathrm{GHz}$. From this signal the upper limit of locked laser line width can be estimated.

The primary configuration of our laser system is a master-slave scheme in which ECDL2 is PDH-locked to the Cav2 cavity and ECDL1 is phase-locked to ECDL2. This configuration allows for wide frequency tuning with narrow line width of ECDL1 making it useful to very 
high resolution spectroscopy. After ECDL2 is locked to its cavity the beat note signal (RF1) which is equal to frequency difference between two lasers is mixed with signal (LO1) from generator (Gen1) to down-convert it to about $20 \mathrm{MHz}$ (see Fig. 7b). Next, this signal (RF2) is mixed with $20 \mathrm{MHz}$ signal (LO2) from a second generator (Gen2). The low-frequency output of Mixer 2 is proportional to the phase difference between both input signals and serves as an error signal for the phase lock of ECDL1 to ECDL2. Response to the error signal, produced by FALC, controls the laser diode current. This single-stage feedback loop is able to phase-lock the laser, however it is relatively easy to break the lock by for example hitting the optical table. This is because linearity of the error signal produced by mixer is limited to phase difference of $\pm \pi$ so any phase perturbation higher than this limit breaks the lock.

Therefore the second stage of the feedback loop was added to make system more insensitive to perturbations. The phase-frequency detector (PFD) placed in parallel with Mixer 2 generates an error signal proportional to the phase difference between the two lasers. The PFD extends the range of linearity of the error signal with phase difference from $\pm \pi$ to $\pm 128 \pi$. PFD used here is similar to that presented in Ref. [30], where its principle of operation is described. Comparing to a mixer the PFD response to phase difference in the inputs is slower but range of linear response is much bigger. The error signal produced by PFD is connected to another PID controller (Vescent Photonics D2-125, with $10 \mathrm{MHz}$ maximum bandwidth) and its response controls the current of ECDL1 diode by the second current modulation input of the laser head. This stage of the feedback loop allows to automatically recapture the phase lock after incidental perturbation of relative phase of RF2 and LO2 signals over the range of linear response of mixer $( \pm \pi)$. While both the mixer stage and the PFD stage of feedback loop control the same diode current, they do not perturb each other and laser lock is stable for hours.

Once ECDL1 is locked to ECDL2, its relative tuning is realized by changing frequency of LO1 signal from Gen1 generator. Signal RF2 is kept equal to LO2 by the feedback loop which tunes the laser frequency difference exactly as much as LO1 signal was tuned. Due to limited bandwidth of Mixer1 currently maximum tuning range of laser is about $400 \mathrm{MHz}$. In principle $20 \mathrm{GHz}$ tuning range (limited by beat note detector bandwidth and Gen1 generator) could be achieved by using sufficiently fast mixer. It is worth noting that frequencies of RF2 and LO2 signals do not change while laser is tuning and can be set to value optimal for PFD operation.

\section{Results and discussion}

In order to measure line width of our laser PDH-locked to the optical cavity both lasers ECDL1 and ECDL2 were locked to their cavities with frequency difference between both lasers of $270 \mathrm{MHz}$. By measurement of a power spectrum of the beat note signal a line width of lasers can be estimated. In Fig. 8 power spectrum of the beat note of both locked laser beams are presented. In the main plot (a) frequency span of the spectrum analyzer (Agilent E4440A) was set to $950 \mathrm{~Hz}$, resolution bandwidth was $9 \mathrm{~Hz}$, and sweep time was $0.2 \mathrm{~s}$. Assuming the Lorentzian shape of of the beat note signal from the least-squares fit we obtained full width at half maximum (FWHM) of $(16.8 \pm 0.2) \mathrm{Hz}$. Assuming that measured beat note is a convolution of two Lorentzian distributions of both laser spectra, the average FWHM of one laser is $8.4 \mathrm{~Hz}$. In the inset (b) of Fig. 8 the same beat note signal is shown as in Fig. 8a but measured at wider frequency span of $15 \mathrm{MHz}$. Except of central peak (broadened because of spectrum analyzer resolution decrease) characteristic broad peaks occur at frequencies of about $\pm 1.5 \mathrm{MHz}$ from the central peak. This frequency corresponds to the bandwidth of the laser-lock feedback loop, see e.g. [31].

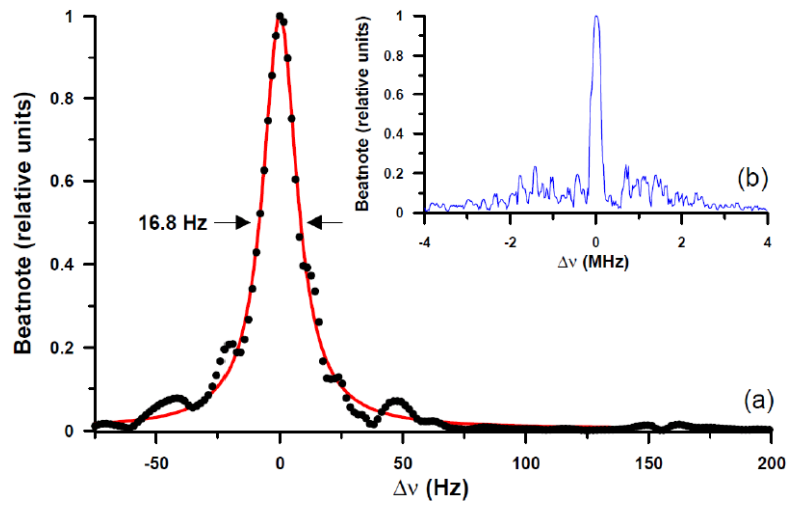

Fig. 8. Power spectrum of the beat note of two independently locked lasers: (a) resolution bandwidth $9 \mathrm{~Hz}$, (b) resolution bandwidth $150 \mathrm{kHz}$.

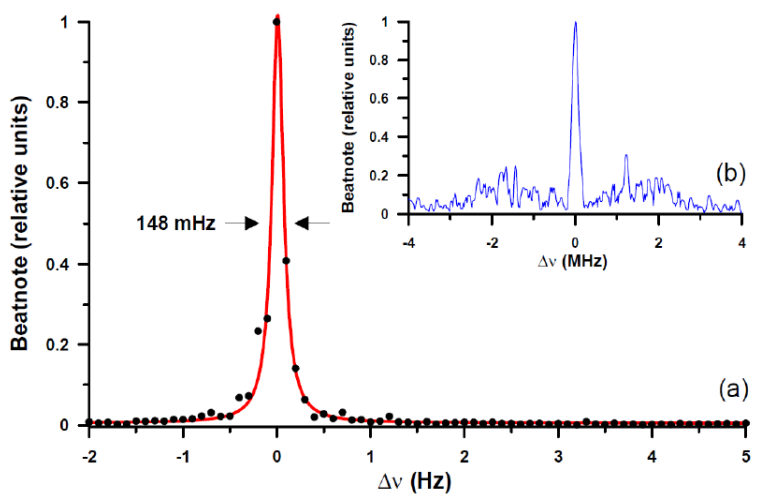

Fig. 9. Power spectrum of the beat note of two phase-locked lasers: (a) from FFT of $10 \mathrm{~s}$ waveform, (b) from spectrum analyzer with resolution bandwidth $110 \mathrm{kHz}$.

Measurement of quality of the phase locking of ECDL1 to ECDL2 requires different approach than that presented for two independent lasers because the beat note 
signal line width is smaller than resolution of our spectrum analyzer. For this reason beat note signal (RF2 signal in Fig. 7) was down-converted to $600 \mathrm{~Hz}$ with use of additional ZP-3 mixer and signal generator. This low-frequency signal was measured in $10 \mathrm{~s}$ time period and power spectrum was calculated from FFT. In Fig. 9a this beat note signal spectrum is presented. From least-squares fit of the Lorentzian profile estimated FWHM is $(148 \pm 7) \mathrm{mHz}$. In the inset (b) of Fig. 9 a beat note signal of relative laser lock is shown in wider frequency span measured by spectrum analyzer.

As for PDH lock of laser to the cavity, also for the phase lock the bandwidth limit estimated from two sidebands of beat note spectrum is about $1.5 \mathrm{MHz}$. This result agree with a fact that the same feedback loop filter (Toptica FALC) was used for both locking schemes.

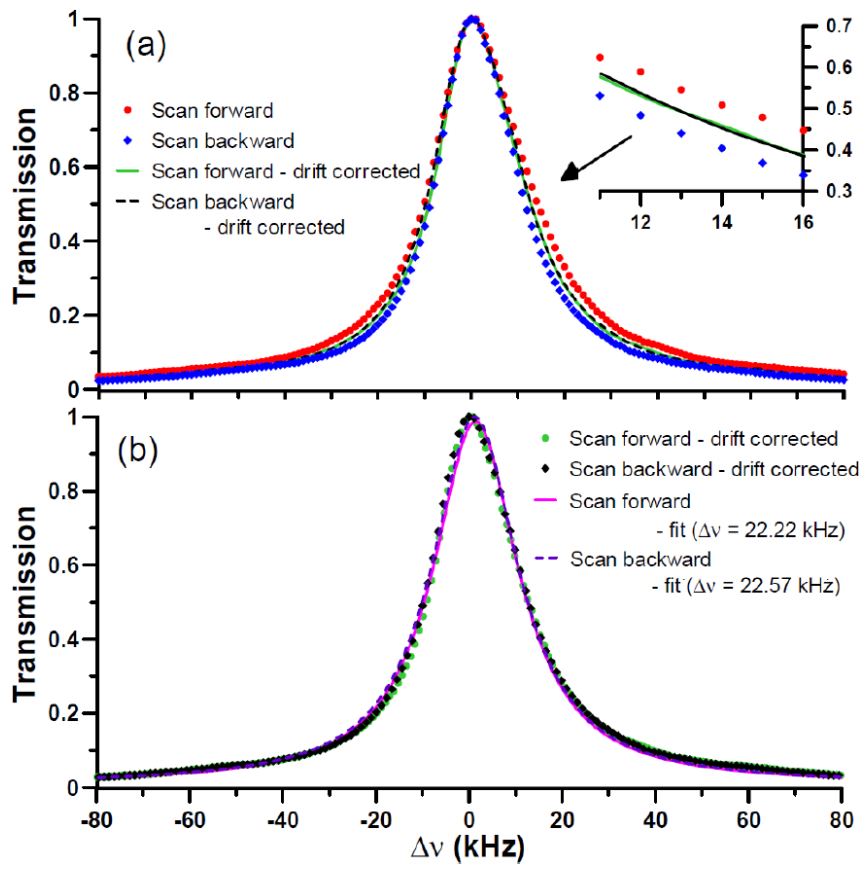

Fig. 10. Transmission spectrum of $\mathrm{TEM}_{00}$ mode of Cav1 cavity: (a) direct measurement, (b) after laser frequency drift correction.

As a test of applicability of our stable laser system to high-resolution spectroscopy we measured transmission spectrum of Cav1 cavity near one of its $\mathrm{TEM}_{00}$ modes. In Fig. 10a two such transmission spectra are presented. Two scans differ by frequency tune direction. Transmission peak measured by scanning laser forward is clearly broader than the second one made with scan backward. This difference is caused by slow frequency drift of Cav1 with respect to Cav2 modes to which our laser is locked. It is mainly associated with unequal temperature drift of both cavities. Since temperature drift rate vary slowly in time for a period of one spectrum measurement $(200 \mathrm{~s})$ we assume constant $\mathrm{d} \nu / \mathrm{d} t$ drift. Frequency drift was measured by instantaneously detuning of the laser frequency from Cav1 cavity resonance by $100 \mathrm{kHz}$ and measuring

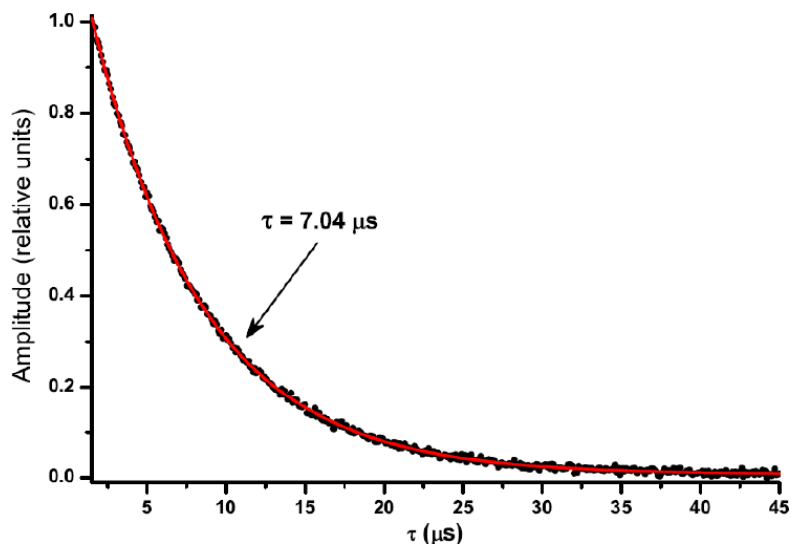

Fig. 11. Ring-down signal from Cav1 cavity with fitted exponential decay.

time after which laser drifts back to the mode center. We obtained $\mathrm{d} \nu / \mathrm{d} t=(300 \pm 9) \mathrm{Hz} / \mathrm{s}$. After applying this correction to our spectra their shape became very similar. Corrected spectra together with fitted Lorentzian profiles are presented in Fig. 10b. Fitted widths (FWHM) of measured cavity mode $\Delta \nu_{\mathrm{c}}$ are $(22.22 \pm 0.10) \mathrm{kHz}$ and $(22.57 \pm 0.09) \mathrm{kHz}$ for scans forward and backward, respectively. This small difference can be explained by uncertainty of measured laser frequency drift. We should note that measured cavity mode profile is slightly asymmetrical, which may be caused by non-ideal mode matching of the laser beam to the cavity $\mathrm{TEM}_{00}$ mode.

Width of the Cav1 cavity mode $\Delta \nu_{\mathrm{c}}$ can be verified independently by measurement of the time constant $\tau$ of the cavity ring-down (CRD) decay signal. In Fig. 11 measured signal of the CRD decay is presented together with fitted exponential function. Switching off the laser beam was realized by acousto-optic modulator (AOM) with a fast driver providing switching off time below 100 ns. As seen in Fig. 11, signal is single exponential. From the average of 6 measured time constants $\langle\tau\rangle=7.050 \pm 0.018 \mu \mathrm{s}$ the cavity mirrors reflectivity $R$ can be calculated

$$
R=\exp \left(-\frac{L}{c \tau}\right) \approx 1-\frac{L}{c \tau},
$$

where $L$ is the cavity length and $c$ is the speed of light. For our cavity with $L=10 \mathrm{~cm}$ mirrors reflectivity calculated from measured $\tau$ is $R=0.99995269$ (13) and the cavity mode width is

$$
\Delta \nu_{\mathrm{c}}=\frac{\nu_{\mathrm{f}}}{F^{*}}=\frac{\nu_{\mathrm{f}}(1-R)}{\pi \sqrt{R}} .
$$

From this equation cavity mode width measured by CRDS method is $\nu_{\mathrm{c}}=(22.58 \pm 0.07) \mathrm{kHz}$. Here we should note that reflectivity $R$ is a very broadband feature of mirrors and in a sub-MHz range of frequency can be treated as constant. Therefore mode width $\Delta \nu_{\mathrm{c}}$ determined from CRDS is much less dependent on laser line width than mode width measured by direct transmission spectroscopy, for which a convolution of cavity mode and laser instrumental function should be observed in trans- 
mission signal. Good agreement between $\nu_{\mathrm{c}}$ measured from CRDS and from transmission spectrum clearly indicates negligible width of laser instrumental function for application to $\mathrm{kHz}$ level spectroscopy.

\section{Conclusions}

Our laser system achieved $8 \mathrm{~Hz}$ level of short term stability. The main limitation in the current state of this setup is instability of temperature of the optical cavity. Taking into account that only passive stabilization of temperature is applied we should achieve further laser line narrowing by adding active stabilization of the vacuum chamber in which the cavity is installed. Considering application of laser as a local oscillator for $\mathrm{Sr}$ optical clock its systematical drift of frequency should be reduced by at least a factor of 100 to achieve $\mathrm{d} \nu_{\mathrm{c}} / \mathrm{d} t$ rate of the order $1 \mathrm{~Hz} / \mathrm{s}$. Then a sub $\mathrm{Hz}$ spectroscopy of ultra-narrow Sr clock transition can be realized. Taking into account that currently temperature of the vacuum chamber changes by an order of $\mathrm{K}$ per day its active stabilization to a few $\mathrm{mK}$ should be sufficient. Additionally with active stabilization temperature of the cavity can be set closer to a point at which coefficient of thermal expansion of the ULE glass crosses zero and make temperature-induced frequency drift even smaller.

Presented laser system is fully applicable to a $\mathrm{kHz}$ level spectroscopy, as was confirmed by measurements of $22.5 \mathrm{kHz}$ wide optical cavity mode. No influence of laser instrumental function was observed in experimental data. Laser system will be used for further Doppler-free investigation of line shape effects of the ${ }^{3} P_{1}-{ }^{1} S_{0}$ Sr transition which has natural line width below $7 \mathrm{kHz}$ and was investigated in Ref. [32].

Another planned application of our stable laser is ultra-high precision spectroscopy of oxygen $B$-band transitions. Our recent progress in CRDS [21, 22] allowed to increase signal-to-noise ratio of absorption axis of the spectrum to $10^{4}$ level [21]. At this point a factor that limits precision of line shape measurements is stability of the frequency axis which is as good as the reference laser to which the ring-down cavity is locked (currently a $\mathrm{He}-\mathrm{Ne}$ laser with frequency stability of $1 \mathrm{MHz}$ ). Use of our ultra-narrow laser system as a frequency reference for CRDS should dramatically improve its precision. Moreover laser with a $\mathrm{Hz}$ level line width allows for cavity-enhanced spectroscopy of oxygen with resolution higher than natural limitation of CRDS associated with width of the cavity resonance.

As has been recently demonstrated [33], weak oxygen transitions near $687 \mathrm{~nm}$ are very promising with respect to precise determination of gas temperature from the Doppler width. Their application to optical determination of the Boltzmann constant requires relative accuracy of $10^{-6}$ of the Doppler width determination [34-36]. For about $1 \mathrm{GHz}$ Doppler width of these transitions at temperature of $0^{\circ} \mathrm{C}$ required stability of frequency axis must be about $1 \mathrm{kHz}$. Such short- and long-term stabil- ity should be easily provided by our laser system after it is locked to $\mathrm{Sr}$ transition.

\section{Acknowledgments}

The research is part of the program of the National Laboratory FAMO in Torun, Poland and was supported by the Polish MNiSW project No. N N202 148933 and the Foundation for Polish Science TEAM Project cofinanced by the EU European Regional Development Fund.

\section{References}

[1] C. Salomon, D. Hils, J.L. Hall, J. Opt. Soc. Am. B 5, 1576 (1988).

[2] B.C. Young, F.C. Cruz, W.M. Itano, J.C. Bergquist, Phys. Rev. Lett. 82, 3799 (1999).

[3] T. Nazarova, F. Riehle, U. Sterr, Appl. Phys. B 83, 531 (2006).

[4] L. Chen, J.L. Hall, J. Ye, Phys. Rev. A 74, 053801 (2006).

[5] S.A. Webster, M. Oxborrow, P. Gill, Phys. Rev. A 75, 011801(R) (2007)

[6] J. Millo, D.V. Magalhães, C. Mandache, Y. Le Coq, E.M.L. English, P.G. Westergaard, J. Lodewyck, S. Bize, P. Lemonde, G. Santarelli, Phys. Rev. A $\mathbf{7 9}$ 053829 (2009).

[7] M. Notcutt, L.-S. Ma, J. Ye, J.L. Hall, Opt. Lett. 30 $1815(2005)$

[8] H. Stoehr, F. Mensing, J. Helmcke, U. Sterr, Opt. Lett. 31, 736 (2006).

[9] A.D. Ludlow, X. Huang, M. Notcutt, T. Zanon-Willette, S.M. Foreman, M.M. Boyd, S. Blatt, J. Ye, Opt. Lett. 32, 641 (2007).

[10] P. Dubé, A.A. Madej, J.E. Bernard, L. Marmet, A.D. Shiner, Appl. Phys. B 95, 43 (2009).

[11] T. Ido, H. Katori, Phys. Rev. Lett. 91, 053001 (2003).

[12] T. Ido, T.H. Loftus, M.M. Boyd, A.D. Ludlow, K.W. Holman, J. Ye, Phys. Rev. Lett. 94, 153001 (2005).

[13] T. Zelevinsky, M.M. Boyd, A.D. Ludlow, T. Ido, J. Ye, R. Ciuryło, P. Naidon, P.S. Julienne, Phys. Rev. Lett. 96, 203201 (2006).

[14] Y.N. Martinez de Escobar, P.G.Mickelson, P. Pellegrini, S.B. Nagel, A. Traverso, M. Yan, R. Côté, T.C. Killian, Phys. Rev. A 78, 062708 (2008).

[15] S.A. Diddams, J.C. Bergquist, S.R. Jefferts, C.W. Oates, Science 306, 1318 (2004).

[16] M.M. Boyd, A.D. Ludlow, S. Blatt, S.M. Foreman, T. Ido, T. Zelevinsky, J. Ye, Phys. Rev. Lett. 98 , 083002 (2007).

[17] T. Rosenband, D.B. Hume, P.O. Schmidt, C.W. Chou, A. Brusch, L. Lorini, W.H. Oskay, R.E. Drullinger, T.M. Fortier, J.E. Stalnaker, S.A. Diddams, W.C. Swann, N.R. Newbury, W.M. Itano, D.J. Wineland, J.C. Bergquist, Science 319, 1808 (2008). 
[18] A.D. Ludlow, T. Zelevinsky, G.K. Campbell, S. Blatt, M.M. Boyd, M.H.G. de Miranda, M.J. Martin, J.W. Thomsen, S.M. Foreman, Jun Ye, T.M. Fortier, J.E. Stalnaker, S.A. Diddams, Y. Le Coq, Z.W. Barber, N. Poli, N.D. Lemke, K.M. Beck, C.W. Oates, Science 319, 1805 (2008).

[19] T. Akatsuka, M. Takamoto, H. Katori, Nature Phys. 4, 954 (2008).

[20] J. Lodewyck, P. Westergaard, A. Lecallier, L. Lorini, P. Lemonde, New J. Phys. 12, 065026 (2010).

[21] A. Cygan, D. Lisak, P. Masłowski, K. Bielska, S. Wójtewicz, J. Domysławska, R.S. Trawiński, R. Ciuryło, H. Abe, J.T. Hodges, Rev. Sci. Instrum. 82, 063107 (2011).

[22] S. Wójtewicz, D. Lisak, A. Cygan, J. Domysławska, R.S. Trawiński, R. Ciuryło, Phys. Rev. A 84, 032511 (2011).

[23] H.B. Callen, R.F. Greene, Phys. Rev. 86, 702 (1952).

[24] K. Numata, A. Kemery, J. Kamp, Phys. Rev. Lett. 93, 250602 (2004).

[25] M. Notcutt, L.-S. Ma, A.D. Ludlow, S.M. Foreman, J. Ye, J.L. Hall, Phys. Rev. A 74, 031804(R) (2006).

[26] C. Rathmann, G. Mann, M. Nordberg, Appl. Opt. 7, 819 (1968).

[27] A. Yamaguchi, M. Fujieda, M. Kumagai, H. Hachisu, S. Nagano, Y. Li, T. Ido, T. Takano, M. Takamoto, H. Katori, Appl. Phys. Expr. 4, 082203 (2011).
[28] R.W.P. Drever, J.L. Hall, F.V. Kowalski, J. Hough, G.M. Ford, A.J. Munley, H. Ward, Appl. Phys. B 31, 97 (1983).

[29] E.D. Black, Am. J. Phys. 69, 79 (2000).

[30] M. Prevedelli, T. Freegarde, T.W. Hansch, Appl. Phys. B 60, S241 (1995).

[31] R.W. Fox, C.W. Oates, L.W. Hollberg, in: Cavity Enhanced Spectroscopies, Eds. R.D. van Zee, J.P. Looney, Academic Press, 2003, p. 32.

[32] N. Shiga, Y. Li, H. Ito, S. Nagano, T. Ido, K. Bielska, R. Trawiński, R. Ciuryło, Phys. Rev. A 80, 030501(R) (2009).

[33] A. Cygan, D. Lisak, R.S. Trawiński, R. Ciuryło, Phys. Rev. A 82, 032515 (2010).

[34] K.M.T. Yamada, A. Onae, F.-L. Hong, H. Inaba, T. Shimizu, C.R. Phys. 10, 907 (2009).

[35] C. Lemarchand, M. Triki, B. Darquié, Ch.J. Bordé, C. Chardonnet, C. Daussy, New J. Phys. 13, 073028 (2011).

[36] A. Castrillo, M.D. De Vizia, L. Moretti, G. Galzerano, P. Laporta, A. Merlone, L. Gianfrani, Phys. Rev. A 84, 032510 (2011). 